\title{
Indicadores de productividad, colaboración y circulación de la investigación. Caso: Escuela de Bibliotecología y Archivología de Luz
}

\author{
Dionnys PEÑA OCANDO \\ Escuela de Bibliotecología y Archivología. Universidad del Zulia. Venezuela \\ dionnysp@gmail.com \\ Lisbeth PORTILLO \\ Escuela de Bibliotecología y Archivología. Universidad del Zulia. Venezuela \\ liportil@gmail.com \\ Edixson CALDERA MORILLO \\ Escuela de Bibliotecología y Archivología. Universidad del Zulia. Venezuela \\ ejocal@yahoo.es
}

Recibido: 31/03/2011

Aceptado: 4/05/2011

\section{RESUMEN}

El presente artículo comprende el análisis del desempeño investigativo de la Escuela de Bibliotecología y Archivología de la Universidad del Zulia con base en algunos indicadores de productividad, colaboración y circulación. Como metodología se utilizaron técnicas bibliométricas basadas en la recolección de los datos obtenidos a través de diversas fuentes de información (expedientes y currículos de los profesores) y bases de datos en línea (portales, sitios web, blog, entre otros). Los resultados arrojan un crecimiento paulatino en la productividad de algunos investigadores, el estado del arte en cuanto a la autoría, coautoría y tutorías en las investigaciones, además de su visibilidad en los distintos medios de difusión científica. Se concluye que aunque la productividad científica en esta escuela se encuentra en crecimiento y la colaboración intrainstitucional de los investigadores se ha venido fortaleciendo, aún necesitan resolverse las limitaciones de publicación, almacenamiento y flujo de la producción científica en Ciencias de la Información en la bases de datos locales para incrementar su visibilidad a través de la socialización del conocimiento.

Palabras Clave: Productividad científica, colaboración de los autores, circulación de la investigación, indicadores bibliométricos, Escuela de Bibliotecología y Archivología.

Indicators of Productivity, Collaboration and Circulation of the Investigation. Case: School of Library and Archive of the University of Zulia

\begin{abstract}
The present article covers the analysis of the investigative performance of the School of Library and Archives in the University of Zulia, base on some indicators of productivity, collaboration and circulation. As methodology, it was used the bibliometric techniques based on the collection of the data obtained through different sources of information (files and curriculum of professors)
\end{abstract}


and on line data bases (portals, websites, and blog, among others). The results show a gradual growth in the productivity of some investigators, the state of the art related to the author, coauthor and tutoring in the investigations, as well as its visibility in the different media of scientific diffusion. It is conclude, that although the scientific productivity in this school is growing and the intra-institutional collaboration of investigators has been strengthened, still it is need to resolve the limitations of publication, storage and flow of scientific production in Information Science in the local data bases to increase its visibility through the socialization of knowledge.

Keywords: Scientific productivity, collaboration of the authors, circulation of the investigation, bibliometric indicators, School of Library and archives.

\section{INTRODUCCIÓN}

Esta primera década del siglo XXI sigue siendo testigo de los problemas que tradicionalmente han venido aquejando a la investigación latinoamericana en todos los campos del saber y en los diversos sectores de producción o generación científica en Latinoamérica. Son pocas las reflexiones sobre las actividades investigativas donde no se termine por plantear las anomalías presentes en variables que afectan su calidad y pertinencia, impidiendo que ésta logre impactar significativamente a la región en pro de su desarrollo.

En el caso Venezolano, aún cuando se reconoce que la calidad de la producción científica ha venido mejorando en las últimas décadas, Finol (2008), Márquez (2006), sostienen que se requieren de estudios de evaluación que permitan descifrar con claridad su impacto y crecimiento.

Dado el escenario anterior, la evaluación de la investigación comienza a adquirir especial relevancia Milanés y otros (2008), sobre todo en áreas vitales para el desarrollo de la ciencia como es el caso de la información/documentación, la cual provee las herramientas y técnicas para monitorear la producción de conocimientos, pero pocas veces se ha sometido si misma a este riguroso proceso, con miras a diagnosticar empíricamente sus avances científicos y tomar decisiones que atañen a las políticas científicas particulares. Esto supone que en el campo de las Ciencias de Ia información (CI) al igual que en otras áreas del saber, se requiere discernir sobre la calidad y pertinencia del trabajo investigativo.

Son muchas las dimensiones o puntos que pueden ser objeto de evaluación científica, mas para las CI, el análisis de su producción científica, el comportamiento de sus investigadores y la visibilidad de los resultados, siguen siendo temas centrales, más aún en una disciplina que lucha por posicionarse epistemológicamente y por ser reconocida internacionalmente en sus avances. Esto conlleva a la idea de que no sólo basta con producir investigación en información, sino de evaluar objetivamente sus resultados en aras de reorientarlos o reconocer sus aciertos, entendiendo que solo que se evalúa se puede mejorar.

De allí la relevancia de este trabajo en el cual nos hemos propuesto, caracterizar el desempeño investigativo de la Escuela de Bibliotecología y Archivología de la Universidad del Zulia-Venezuela, durante el período 1962- 
2007, a partir de los indicadores de productividad, colaboración y circulación de su producción científica, como complemento de una investigación previa centrada en aspectos cualitativos y cuantitativos de la actividad científica en este contexto académico. Peña y otros, (2009).

Para el logro del fin establecido, se acudió a un estudio bibliométrico de tipo institucional o de grupo de agentes científicos, mediante el uso de indicadores simples con miras a adentrarnos en especificidades propias de un centro de investigación de tradición como que además se considera representativo del país en la materia. Con ello se busca develar problemas medulares que impiden un impacto significativo de la investigación en el área bajo la premisa plateada por Cañedo (2005) de que a pesar de los obstáculos, la reducción de fondos, la carencia de tiempo así como la falta de entrenamiento e interés del personal, en los países latinoamericanos se ha impulsado considerablemente la investigación en el sector y aún existe un cúmulo de conocimientos de gran relevancia que por diversas razones no poseen la visibilidad esperada. Con el producto de este trabajo podrían generarse algunas directrices y esquemas que permitan la organización y la racionalidad del quehacer investigativo, al mismo tiempo que se incremente la productividad científica, bajo el esquema de un mayor aprovechamiento de los recursos destinados para tal fin en la institución.

\section{INDICADORES BIBLIOMÉTRICOS PARA EVALUAR LAS CIENCIAS DE LA INFORMACIÓN}

Es conocido, que las Ciencias de la Información brinda las técnicas e instrumentos para medir la producción de conocimientos y su transformación en bienes dentro del sistema científico general, mediante disciplinas como la bibliometría, cienciometría e informetría. Arecibia y Moya, (2008). Por ello resultaría paradójico no acometer estudios evaluativos usando estos recursos con su propia producción, lo cual supone que la evaluación es un marco general en el que pueden entrar todas las disciplinas, inclusive las informativas.

Ya muchos estudios tradicionales se han realizado en el tema de la evaluación científica aplicada a las CI recurriendo a la bibliometría como herramienta metodológica1, la cual permite la medición del conocimiento científico producido y registrado, a través de la aplicación de tratamientos cuantitativos y estadísticos, examinando las relaciones entre diferentes variables: recursos humanos-documentos, artículos-periódicos, producción-consumo, etc., (Vanti, 2000; Dávila 2009).

A su vez, para lograr los fines de medición del conocimiento producido, la bibliometria se vale de indicadores que se configuran como rasgos que explican el comportamiento de la ciencia y la tecnología, los cuales son definidos por Maltrás

\footnotetext{
${ }^{1}$ Tal es el caso de los trabajos de Gorbea-Portal (2001); Licea de Arenas y otros (2000); HernándezSalazar (2005); Herrero- Solana y Rios-Gómez (2006), para América Latina, además de las experiencias de Moya-Anegón (2000); Jimenez-Contreras (2000) en el contexto español por citar algunos.
} 
(2003), como medidas obtenidas a partir del análisis estadístico de los rasgos cuantificables de la literatura científica, su importancia radica en ser instrumentos básicos mediante los cuales la sociedad puede acceder a un conocimiento sobre la estructura real de la ciencia y, en su caso, orientar en lo posible la dirección del desarrollo del conocimiento, además de su empleo para el diseño de políticas científicas con vistas a la evaluación de los sistemas de investigación.

El inventario de indicadores bibliométricos ha crecido considerablemente en la última década, lo cual brinda nuevas y nutridas opciones para examinar el comportamiento de la labor científica de la ciencia en general, permitiendo armar baterías de ellos para examinar el comportamiento de un sistema científico particular. Dentro de las clasificaciones más sencillas se encuentran la de Ruiz (2005) y la de Arencibia y Moya (2008) quienes citando a Spinak y Vinkler, los separan en dos grupos: los que miden la calidad y el impacto de las publicaciones científicas (indicadores de publicación), y aquellos que miden la cantidad y el impacto de las vinculaciones o relaciones entre las publicaciones científicas (indicadores de citación).

Arencibia y Moya (2008), también recurren a la división que hacen autores como Bailón-Moreno y Guzmán, Sánchez, quienes los plantean como indicadores de actividad e indicadores relacionales de primera, segunda y tercera generación, siendo los primeros aquellos que proporcionan datos sobre el volumen y el impacto de las actividades de investigación, mediante simples recuentos de elementos bibliográficos (como autores, artículos, palabras clave, patentes, citaciones, entre otros), mientras que los indicadores relacionales se proponen conocer los vínculos y las interacciones entre los diferentes elementos bibliográficos, mediante los conceptos de cocitación y coocurrencia, describiendo el contenido de las actividades y su evolución.

Esto supone dos niveles para los estudios bibliométricos: uno básico-diagnóstico y uno relacional- inferencial, este último más profundo a partir de los cuales se puede predecir y deducir hallazgos mucho mas relevantes que los anteriores. Para una disciplina naciente como la $\mathrm{CI}$, donde la producción científica es aún incipiente y la presencia de fuentes de datos limitada, parece conveniente acudir al primer nivel de indicadores que reflejen el estado de la actividad científica en este campo, de modo tal que pueda conformarse la base para estudios relacionales posteriores.

Ahora bien, las dos tipologías anteriores se complementan con la propuesta de Maltrás (2003), quien sugiere la existencia de tres grupos de indicadores referidos a la producción, la calidad y la colaboración, basado en la idea de Moravcsick y Velho sobre los tres aspectos de la ciencia que pueden ser medidos: actividad, productividad y progreso. De aquí se entiende que los indicadores de producción se encuentran muy relacionados con los recuentos descriptivos de los resultados obtenidos por las actividades de investigación, pero también con la productividad de los investigadores medida según la literatura que estos producen.

De acuerdo con Maltrás, los indicadores de calidad están referidos al impacto de las publicaciones discriminando su importancia e interés para la comunidad científica expresados por medio de las citas recibidas, mientras que los indicadores de colaboración, se entienden como los que informan acerca de las relaciones entre los productores o agentes científicos en el proceso que ha concluido con la publicación 
conjunta de resultados científicos. Este último tipo de indicadores hacen referencia a los aspectos concernientes a los científicos con relación a: el problema de la autoría, la productividad de los autores, el impacto de sus ideas, las relaciones con otros investigadores o grupos de investigación entre otros. López-Yépez, (1999)

Cabe destacar la propuesta de López-Yépez (1999); López-Yépez y Prat Sedeño (2002), sobre los indicadores de circulación, dispersión y obsolescencia relacionados con los mensajes científicos elaborados por los investigadores y a su transmisión y mantenimiento de vigencia; de dónde interesan para fines de este trabajo los primeros, los cuales consideran parámetros como: los canales de comunicación empleados, presencia en las bases de datos y la presencia de artículos en revistas; esto en razón de que los resultados científicos deben circular por canales formales que hagan posible la socialización del conocimiento entre la comunidad científica.

Dichos canales formales son por excelencia las publicaciones científicas pero son las fuentes electrónicas disponibles en bases de datos y sistemas informáticos aquellas que se transforman en espacios fértiles para determinar cómo se usa y se divulga el conocimiento que se genera a través de la investigación o qué tipo de información generamos en nuestra disciplina mediante el "flujo transfronterizo de datos" Morales Citado por Cordova, (2005:94) que permite que las comunidades académicas y sociales en su mayoría, conozcan la producción científica que se está generando en diferentes partes del mundo en el área de la CI.

De modo que la atención de la evaluación bibliométrica en esta disciplina también se encuentra dirigida hacia estos medios, sean de alcance local, regional e internacional, ya que se configuran como la materia prima central para emprender la medición de resultados en el área, bajo una perspectiva cooperativa, interconectada y organizada para lograr el flujo coordinado de datos entre los diversos sistemas y que a la postre se traduce en ventajas de "Visibilidad" de los investigadores y sus productos científicos en las respectivas comunidades y hacia otras con mayores niveles de exigencia.

\section{METODOLOGÍA EMPLEADA}

La medición del desempeño investigativo en el caso estudiado, se tipifica como un estudio bibliométrico, por cuanto se basó en los datos aportados por la producción científica de los integrantes de la comunidad científica de la unidad académica o "grupo de agentes científicos", a través del uso de diversas fuentes de datos tales como:

a) La base de datos del observatorio de ciencia y tecnología disponible en línea mediante la siguiente dirección: www.oncti.gob.ve ${ }_{2}$ correspondiente a los investigadores adscritos al programa de promoción al investigador (PPI).

b) Los currículos de los profesores no registrados en la base de datos anteriormente indicada.

c) Los expedientes de los profesores jubilados de la escuela que formaron parte de la planta profesoral durante sus primeros años de funcionamiento, cu- 
ya información fue obtenida a través del Centro de Documentación, Informacón y Archivo de la Universidad del Zulia (CEDIA).

d) Las bases de datos en línea (Scopus, Dialnet, e-libro, Scielo y Revencit), los catálogos automatizados de las principales universidades públicas y privadas de Venezuela y la búsqueda libre en la Web (local, nacional e internacional) mediante el buscador Google. Esta herramienta permitió obtener registros de la visibilidad de los profesores en función de su aparición en eventos, publicaciones, citaciones y demás participaciones científicas.

De dichas fuentes, se extrajeron los datos correspondiente a la formación de talento humano (tutoría de investigaciones), trabajos en conferencias y congresos, publicaciones y proyectos de investigación como insumos para dar cuenta de indicadores seleccionados a los fines del presente estudio, tales como la productividad de los autores, la colaboración simple desde la autoría y el flujo de la información científica que circula por los canales formales inmediatos, adicionalmente se decidió estudiar el comportamiento en materia de tutorías como una manera de explorar la forma como se conduce al formación de nuevos investigadores en el área.

La población de investigadores asumida para el estudio fueron los profesores (activos y jubilados) que han conformado la planta profesoral de la Escuela de Bibliotecología y Archivología de la Universidad del Zulia en sus 45 años de funcionamiento, incluyendo aquellos que no pertenecieron directamente a esta entidad pero que realizaron aportes significativos a la docencia y la investigación en el área; dicha población quedó conformada por 28 profesores, la cual es representativa de los productores de investigación en Ciencias de la Información en el estado Zulia, ya que no existe otro centro, instituto o escuela especializada en esta área en la región que se dedique a la labor investigativa.

Este grupo de investigadores aportaron una producción científica de 838 trabajos en el período estudiado. Corpus este que fue analizado desde la perspectiva de los indicadores establecidos y representado mediante medidas de distribución de frecuencias absolutas y relativas. Finalmente cada gráfico fue interpretado con base en los referentes teóricos del estudio a objeto de establecer las comparaciones teoría-realidad, necesarias para evaluar el comportamiento de los indicadores de la producción intelectual.

\section{ANALISIS DE LOS RESULTADOS}

Los hallazgos obtenidos fueron agrupados conforme a los indicadores empleados, mostrando no solo valores cuantitativos sino también aproximándonos a interpretaciones cualitativas que puedan servir para describir y contextualizar cada medida.

\section{Indicadores de productividad}

Este tipo de indicadores plantea un análisis de la productividad por investigador, donde pudo apreciarse según la tabla 1, que el Prof. Johann Pirela aparece como el más 
productivo con 98 trabajos en los que ha intervenido, lo cual representa el 15,58\% de producción total analizada seguido de las profesoras: Egla Ortega, Nelly Primera y Ana Judith Paredes, tomando en cuenta que para el conteo se asumió el método de asignación completa de participación en los trabajos, independientemente de los solapamientos en las coautorías que tuvieron lugar.

\section{Tabla 1}

\section{Productividad por investigador}

\begin{tabular}{|l|c|c|}
\hline Profesores más Productivos & Cantidad & $\mathbf{\%}$ \\
\hline Ana Judith Paredes & 52 & 8,27 \\
\hline Dionnys Peña & 48 & 7,63 \\
\hline Egla Ortega & 60 & 9,54 \\
\hline Johann Pirela & 98 & 15,58 \\
\hline Lisbeth Portillo & 46 & 7,31 \\
\hline Mariela Chavier & 30 & 4,77 \\
\hline Nelly Primera & 59 & 9,38 \\
\hline Norcka Fernández & 32 & 5,09 \\
\hline Ruby Portillo & 36 & 5,72 \\
\hline Tania Peña & 32 & 5,09 \\
\hline & 493 & 78,38 \\
\hline
\end{tabular}

La aparición en diversos trabajos por parte de los actores identificados permite resaltar su reconocimiento dentro del sistema de recompensas de la ciencia al que aspiran los científicos y que no necesariamente tienen que estar vinculados con el incentivo económico sino con su prestigio e intercambio de sus ideas Maltrás, (2003), de modo que con este resultado se develan los responsables de maximizar la labor científica que muchas veces no se han representado en otros medios.

Con relación a los tipos de productos por investigador, se puede observar en la tabla 2, la tendencia hacia un tipo de particular de trabajos abandonando las armonías en algunos binomios: ponencias-artículos, proyectos-ponencias. En consecuencia, es notable el desequilibrio entre la alta cantidad de ponencias presentadas y los pocos proyectos de investigación elaborados o los artículos publicados en consecuencia, es decir, se observa que muchas de las ponencias que se presentan en eventos luego no llegan a convertirse en artículos o en textos publicados. 


\section{Tabla 2}

\section{Producción intelectual por tipo de trabajo}

\begin{tabular}{|l|l|l|l|l|}
\cline { 2 - 5 } \multicolumn{1}{c|}{} & TAE & Ar & Po & Pr \\
\hline Alba Moreno & 4 & 0 & 3 & 0 \\
\hline Alicia López & 2 & 0 & 3 & 0 \\
\hline Alida Molina & 3 & 0 & 0 & 0 \\
\hline Ana Judith Paredes & 2 & 8 & 16 & 26 \\
\hline Aura Franchi & 4 & 0 & 3 & 0 \\
\hline Bertha Cambar & 3 & 0 & 3 & 1 \\
\hline Dionnys Peña & 3 & 9 & 29 & 7 \\
\hline Dunia García & 5 & 0 & 0 & 1 \\
\hline Edixson Caldera & 0 & 4 & 7 & 2 \\
\hline Egla Ortega & 2 & 10 & 26 & 22 \\
\hline Evaristo Arroyo & 2 & 2 & 2 & 2 \\
\hline Evelyn Crespo & 1 & 0 & 1 & 1 \\
\hline Evelyn Graterol & 1 & 1 & 10 & 2 \\
\hline Flora Urdaneta & 3 & 0 & 4 & 0 \\
\hline Francys Delgado & 4 & 2 & 6 & 1 \\
\hline Jodlin Huerta & 7 & 0 & 0 & 0 \\
\hline Johann Pirela & 5 & 31 & 55 & 7 \\
\hline Justa Noemi García & 3 & 0 & 0 & 0 \\
\hline Laura Apitz & 4 & 0 & 0 & 0 \\
\hline Lilia Ferrer & 1 & 2 & 5 & 2 \\
\hline Lisbeth Portillo & 3 & 12 & 26 & 5 \\
\hline María Nelly Santander & 4 & 0 & 0 & 0 \\
\hline Mariela Chavier & 2 & 4 & 21 & 3 \\
\hline Militza Bracho & 3 & 3 & 7 & 2 \\
\hline Nelly Primera & 3 & 13 & 38 & 5 \\
\hline Norcka Fernández & 3 & 6 & 21 & 2 \\
\hline Ruby Portillo & 2 & 3 & 21 & 10 \\
\hline Tania Peña & 6 & 10 & 15 & 1 \\
\hline & 85 & 120 & 322 & 102 \\
\hline
\end{tabular}

TAE: Trabajos académicos especiales

Ar: Artículo

Po: Ponencias

Pr: Proyectos

Tal desequilibrio entre los productos generados por cada investigador, permite traer a colación el planteamiento de algunos autores que abogan por la idea de que la producción científica parta de trabajos con alta rigurosidad científica como las tesis 
doctorales o proyectos de investigación ampliamente desarrollados.Hernández 2005)); (Cañedo, 2005) de modo que exista coherencia entre lo que se produce y lo que se difunde, teniendo presente que el aumento del conocimiento en CI, solo se verá impactado por la cantidad y la calidad de la literatura que se publique.

\section{Indicadores de colaboración simple}

Con respecto a la autoría de la producción cientifica, se observa que aunque el mayor porcentaje de los trabajos $(50,87 \%)$ se continúa realizando en solitario, existe una tendencia interesante hacia la autoría múltiple en el 49,13\% de los trabajos que resultó de sumar los porcentajes de documentos con dos, tres, cuatro, cinco y más de cinco autores según puede verse en la tabla 3. Esto se considera como uno de los signos de la profesionalización del campo de investigación dado que los trabajos con múltiples autores tienen mayor impacto y calidad que los de un solo autor Lawani, citado por Spinak, (1996), dado que se amplía el margen de citas de los mismos.

\section{Tabla 3}

\section{Autorías y grupos de investigación}

\begin{tabular}{|l|l|l|}
\hline Número de Autores & Cantidad & \% \\
\hline 1 Autor & 352 & 50,87 \\
\hline 2 Autores & 163 & 23,55 \\
\hline 3 Autores & 117 & 16,91 \\
\hline 4 Autores & 41 & 5,92 \\
\hline 5 Autores & 9 & 1,30 \\
\hline Más de 5 Autores & 10 & 1,45 \\
\hline & 692 & 100,00 \\
\hline
\end{tabular}

En cuanto a las tutorías como el proceso de formación de futuros investigadores y de acompañamiento para la creación de conocimiento se encuentra que según la tabla 4 el $81,13 \%$ de las tutorías de los profesores corresponden a los trabajos de alumnos de Pregrado, existiendo una menor proporción de tutorías para niveles de maestría, doctorado y otros trabajos especiales.

\section{Tabla 4: Tutorías}

\begin{tabular}{|l|l|l|}
\hline Tipo de Tutoría & Cantidad & \% \\
\hline Trabajo de Grado & 172 & 81,13 \\
\hline Trabajo de Becaría Docente & 1 & 0,47 \\
\hline Tesis de Especialización & 3 & 1,42 \\
\hline Tesis de Maestría & 33 & 15,57 \\
\hline Tesis Doctoral & 2 & 0,94 \\
\hline Investigaciones Libres & 1 & 0,47 \\
\hline & 212 & 100,00 \\
\cline { 2 - 3 }
\end{tabular}


Este resultado puede estar planteando una ausencia de proyección intra y extrainstitucional del profesor para ser seleccionado como tutor en trabajos de mayor envergadura en otros contextos fuera de la escuela, considerando que las tesis doctorales y las de maestría constituyen manifestaciones de calidad por las contribuciones de innovación y originalidad para la disciplina (López Yepes y Prat Sedeño,2002)); (Hernández 2005) con los que se perpetúan las enseñanzas y el legado de los investigadores a las generaciones futuras.

\section{Indicadores de circulación}

Los resultados del análisis de circulación revelan que la presencia de los profesores de la EBA en la WEB se concentra en las páginas de carácter internacional con un $62 \%$ de un total de 465 registros encontrados mediante búsquedas por los nombres de los investigadores, se destaca además un $29 \%$ de apariciones en páginas venezolanas y solo un $9 \%$ en páginas locales (Ver gráfico 1). Esto le otorga una amplia presencia internacional al investigador, además de representar su inserción en la sociedad de la información para luchar contra las desigualdades y carencias en la obtención de contenidos en el área de la CI desde lo que se produce localmente para el mundo (Cordova, 2005)

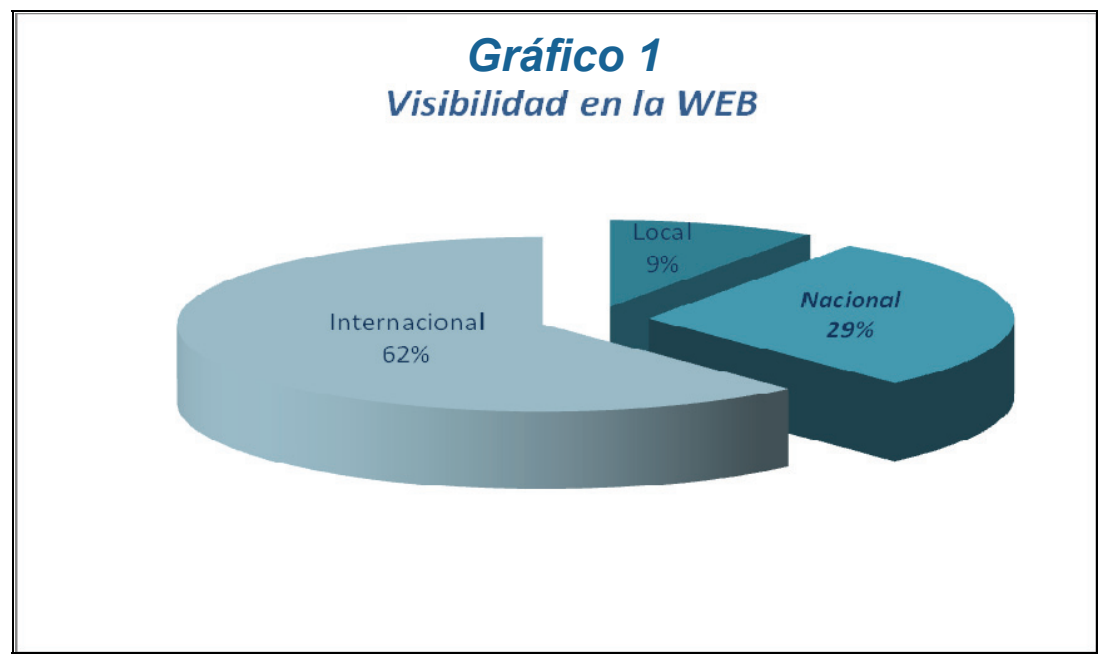

En cuanto a la visibilidad en bases de datos en línea de carácter regional, se observa que es en SCOPUS donde se presenta el mayor porcentaje de apariciones con un 51\%, Dialnet con $26 \%$, Scielo con $19 \%$ y E-libro con $4 \%$; con un total de 57 apariciones en las referidas bases de datos, según se representa en el Gráfico 2. La presencia en estas bases de datos, aunque incipiente en relación con la producción real de la escuela, demuestra que los trabajos de los profesores comienzan a penetrar en sistemas y 
recursos de información especializados que a juicio de Gorbea (2005) permiten compilar la producción científica local y al mismo tiempo implementar indicadores científicos para medir el comportamiento de la misma.

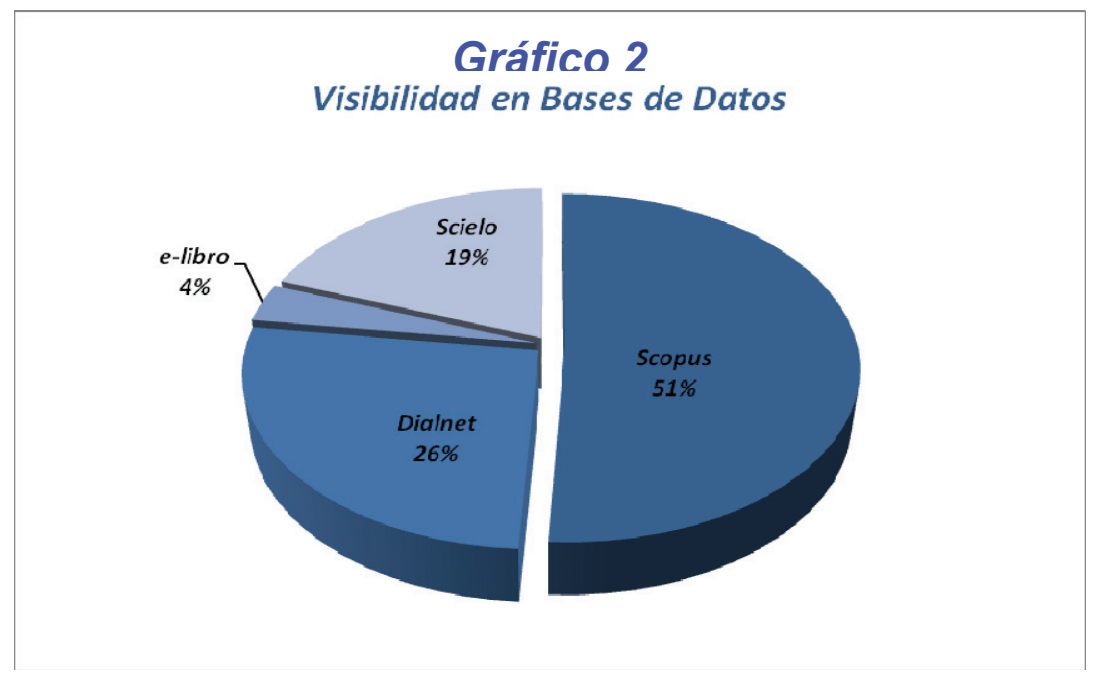

Esto es además un paso importante para la inserción de la producción investigativa de la EBALUZ en diversas bases de datos, a objeto de participar en la compilación del conocimiento generado en esta área en América Latina además, figura como una estrategia cooperativa ante las limitaciones para formar parte de índices internacionales como el Social Science Citatation Index (SSCI), las cuales son consideradas por algunos como muy selectivas y excluyentes.Córdova, (2005) para lograr la visibilidad mundial del conocimiento producido localmente.

La presencia en catálogos públicos de universidades nacionales plantea que el mayor índice de los trabajos se encuentra en la Universidad del Zulia (LUZ) con un $70,11 \%$ y un menor porcentaje en los recursos de la Universidad Rafael Belloso Chacín (URBE), Universidad de los Andes (ULA), Universidad Central de Venezuela (UCV) y Universidad Simón Bolívar (USB), con el 29,89\% de las apariciones, según puede verse en el Gráfico 3.

Esta concentración de investigaciones en el catálogo de LUZ se explica por la cercanía y obligatoriedad de registro de los trabajos de grado, de ascenso y artículos publicados en las revistas de la universidad por parte del Sistema Bibibliotecario y de Información de LUZ, sin embargo esta base de datos no contempla un registro real de la producción realizada por los profesores de la escuela, de manera que existen vacios de trabajos que no quedan almacenados en ningún medio de carácter institucional para la consulta vía web local, nacional o internacional. 


\section{Gráfico 3}

\section{Visibilidad en Catálogos Públicos}

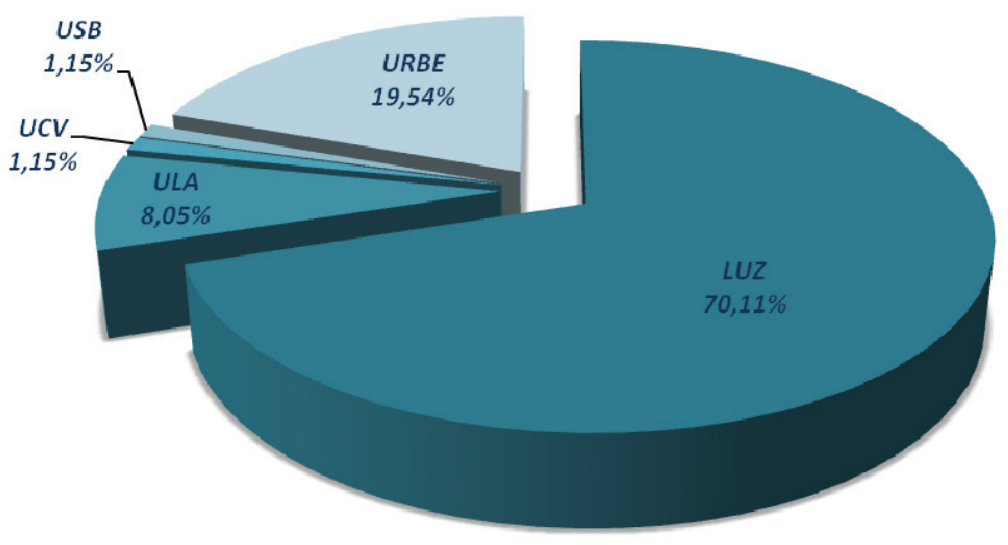

Con relación al medio seleccionado por lo investigadores para la circulación de sus trabajos se destacó la revistas científicas; en tal sentido los resultados muestran una lista de 35 títulos a los cuales se han sometido contribuciones por parte de los investigadores de la escuela, siendo la revista INFOLAC la más seleccionada para exponer los trabajos con $12.79 \%$ de los artículos publicados, seguida de la revista Ciencias Sociales con el 9,30\% de los artículos y Enlace con el 8.14\%, lo cual puede verse en el Gráfico 4 


\section{Gráfico 4 \\ Las diez principales Revistas donde publica la EBALUZ}

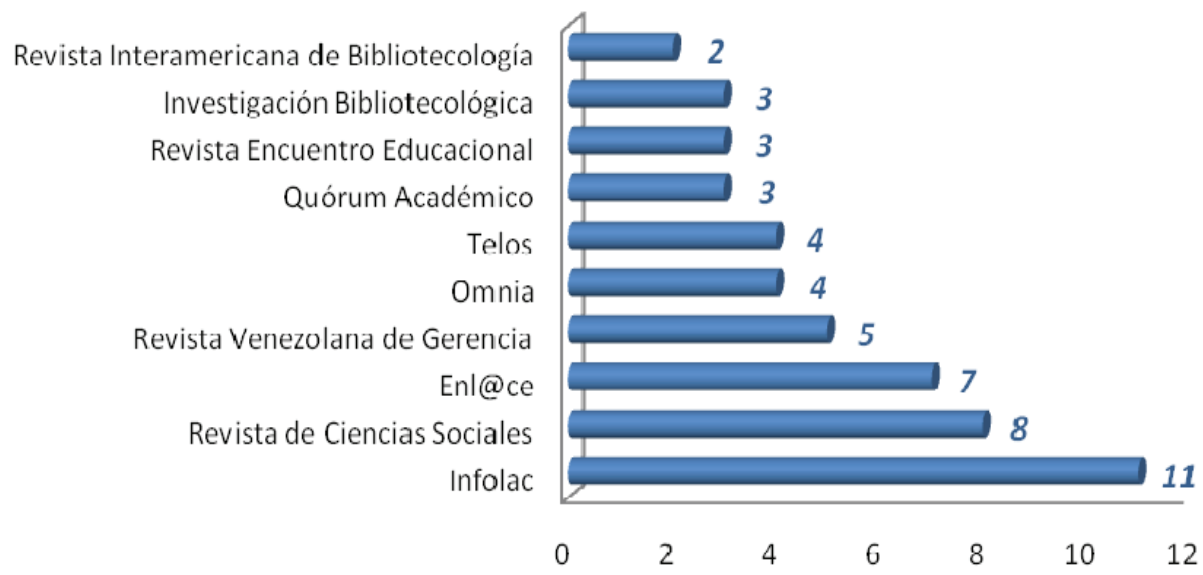

Como puede observarse las revistas elegidas por los investigadores son de carácter nacional ya que éstas son reconocidas como publicaciones de impacto y de prestigio internacional, inclusive reconocidas y avaladas por el Programa de Promoción al investigador y poseen presencia en algunos catálogos regionales. Vessuri (1996), ello en consecuencia ha impedido la visibilidad de esos artículos en el ambito internacional. Requena, (2008)

\section{CONCLUSIONES}

En líneas generales, pudo constatarse que la producción científica de 45 años de la escuela arroja un crecimiento significativo en la productividad de un importante grupo de profesores. Han sido notorios los esfuerzos por producir documentos científicos en diversas modalidades, lo cual ha tenido un uso importante en labores docentes y como referencia obligada para investigaciones locales; de igual manera la generación de documentos en la modalidad de ponencias ha contribuido a posicionar significativamente a algunos investigadores, aun cuando las mismas no han sido transformadas luego en artículos científicos, con serias repercusiones para extender estos trabajos y ser difundidos

Otro factor de gran valor viene representado por el índice de colaboración en labores de investigación, medido para efectos de este trabajo por la coautoría y las tutorías. La primera de ellas revela la tendencia al trabajo en equipo en coautorías múltiples por parte de los miembros de la escuela, lo cual contribuye a fortalecer la investigación y las destrezas compartidas en diversas subáreas de las CI, no obstante el efecto 
multiplicador de la formación con base en las tutorías se ha limitado al pregrado impidiendo una extensión mayor de los conocimientos y descubrimientos en el área.

Por su parte la visibilidad observada a partir de algunos indicadores de circulación, refleja un impacto paradójico de la producción intelectual de los investigadores de la escuela, ya que mientras se observa una importante presencia de los trabajos de los profesores en la WEB y en algunas de las más importantes bases de datos regionales, existe ausencia de los mismos en los catálogos públicos de universidades nacionales. Esto remite a un estudio más profundo de las fuentes de datos nacionales y la vinculación entre las mismas, en virtud de que ello incide negativamente en la visibilidad de nuestra propia producción e impide la posible inserción de importantes trabajos en índices internacionales.

Es deseable para avanzar en el desarrollo científico de la disciplina, llegar a adecuados niveles de visibilidad internacional y regional de nuestra investigación, lo cual pasa por trabajar en la confiabilidad de las bases de datos locales y su vinculación con aquellas de mayor alcance, de igual manera se hace necesario revisar los criterios de divulgación y organización de lo que se produce en materia de ciencias de la información, para ser adecuadamente canalizado hasta el final de la cadena científica.

Finalmente, es importante precisar, que los esfuerzos por publicar se han centrado en revistas nacionales no indizadas en bases de datos de reconocido prestigio internacional, ni en publicaciones en idioma extranjero, barreras éstas que impiden la socialización del conocimiento que se produce desde la escuela de Bibliotecología y Archivología y hace aún incipiente su visibilidad, aunado al hecho de que gran parte de la producción analizada aun no logra formalizarse en artículos científicos pese a su relevancia.

\section{REFERENCIAS DOCUMENTALES}

Arencibia, Jorge, y De Moya Anegón, Félix. La evaluación de la investigación científica: una aproximación teórica desde la cienciometría. ACIMED [online]. 2008, 17/4, pp. 0-0. ISSN 1024-9435.

CAÑEDO ANDALIA, Ruben. Análisis de la evolución histórica y del estado actual de la actividad bibliológico-informacional. Monografia en línea. 2005 Disponible en: http://www.wikilearning.com/analisis_de_la_evolucion_historica_y_del_estado_act ual_de_la_actividad_bibliologico_informacional-wkc-7794.htm. ${ }^{-}{ }^{-}$Consultado ${ }^{-}$el $15 / 01 / 08$.

CÓDOVA, Saray.. El aporte de la base de datos INFOBILA a la investigación costarricense. Ponencia presentada en Seminario

INFOBILA como apoyo a la investigación y Educación en Bibliotecología en América Latina y el Caribe. Compiladores: Filiberto Martínez y Juan Calva.- México: UNAM, Centro Universitario de Investigaciones Bibliotecológicas. 2005 92-107.

DÁVILA, Manuel; Guzman, Roberto y otros. Bibliometría: conceptos y utilidades para el estudio médico y la formación profesional. Salud Uniforme. 2009. 25/2, p. 319330. Disponible en: http://ciruelo.uninorte.edu.co/pdf/salud_uninorte/25- 
2/10_Bibliometria,\%20conceptos\%20y\%20utilidades.pdf. Consultado: $\left[\begin{array}{ll}9 & \text { de }\end{array}\right.$ septiembre de 2009].

FINOL, José E. Calidad y evaluación de la producción científica en Venezuela: Breve análisis y algunas propuestas. Opción. 2006. .22/51, p.131-142. Disponible en: http://www.scielo.org.ve/scielo.php?script=sci_arttext\&pid=S1012-

15872006000300008\&lng=es\&nrm=iso. Consultado: [9 de septiembre de 2009].

Gorbea-Portal, Salvador. Representación bibliométrica del conocimiento latinoamericano en ciencias bibliotecológica y de la información. V Congreso ISKO-España, 2001, 45-53.

GORBEA-PORTAL, Salvador. Proyectos derivados y complementarios de INFOBILA: Índice de citas latinoamericanas y Potencialidades en investigación y docencia iberoamericanas en ciencias bibliotecológica y de la información. Ponencia presentada en Seminario.

HERNÁNDEZ-SALAZAR, Patricia. La investigación bibliotecológica en América Latina: Análisis de su desarrollo. Investigación bibliotecológica. 2005, 20/41, México. 107-140.

HERRERO-SOLANA, Victor y Rios-Gómez, Claudia. (2006). Producción latinoamericana en biblioteconomía y documentación en el Social Cience Citation Index (SSCI) 1966-2003. En IR information research, 2006, 11/2

JIMÉNEZ CONTRERAS, E. La aportación española a la producción científica internacional en biblioteconomía y documentación: balance de diez años (19922001). BiD: Biblioteconomía y Documentación, 2000, (9), 1-29.

LICCA DE ARENAS, J.; Valles, J.; Arévalo, G.; y Cervantes, C. Una visión bibliométrica de la investigación en bibliotecología y ciencia de la información en América latina y El Caribe. Revista Española de Documentación Científica, 2000,23/1, 45-53.

LÓPEZ-YEPES, José y PRTA SEDEÑO, Judith. Propuesta de criterios para la evaluación de la investigación española en Biblioteconomía y documentación: la producción de los científicos y los centros de investigación. Investigación Bibliotecológica. 2002, 16/32. 102-123.

LÓPEZ-YEPES, José. La evaluación de la ciencia en el contexto de las Ciencias de la Documentación. Investigación Bibliotecológica. 1999, 13/27. 195-210.

MALTRAS-BARBA, Bruno Indicadores Bibliométricos. Fundamentos y aplicación al análisis de la ciencia. Asturias: Trea 2003.

MÁRQUEZ F, ÁLVARO B. Calidad y evaluación de la producción científica en Venezuela. Opción. 2006 Disponible en:

$<$ http://redalyc.uaemex.mx/redalyc/src/inicio/ArtPdfRed.jsp?iCve=31022009>

Consultado: [9 de septiembre de 2008].

MILANÉS GUISADO, Yusnelkis; PÉREZ RODRÍGUEZ, Yudit; y otros. Los estudios de evaluación de la ciencia: aproximación teórico métrica. Acimed. 2008 18(6) Disponible en http://bvs.sld.cu/revistas/aci/vol18 6 08/aci041208.htm

MOYA ANEGÓN, F. La investigación española en Recuperación de la Información: análisis bibliométrico (1984-1999). Journal of Spanish Research of Information Science, 2000, 1/1, 117-123. 
PEÑA, Dionnys; CALDERA, Edixson y PORTILLO, Lisbeth. Actividad investigativa en la escuela de Bibliotecología y Archivología de la Universidad del Zulia (19622007): aspectos organizativos y operativos. Revista Ciencias de la información. 2009,.40/3 Septiembre-Diciembre.

REQUENA, Jaime "La visibilidad de nuestra ciencia y técnica. en Revista electrónica Bitacora-e. 2008 (1). Disponible en: http://www.saber.ula.ve/bitstream/123456789/25954/6/articulo14.pdf

RUIZ DE OSMA, Elvira. Evaluación de la ciencia. En Cognosfera "Proyecto de innovación docente "software específico para bibliometría, evaluación de la ciencia y vigilancia tecnológica. 2005

Disponible:http://www.ugr.es/ rruizb/cognosfera/sala_de_estudio/ciencimetria_redes conocimiento/evaluacion_de_la_ciencia.htm. Consultado: 9 de septiembre de 2008].

SPINAK, Ernesto. Diccionario enclopedico de Bibliometría, Cienciometría e informetría. Caracas-Venezuela: UNESCO. 1996, Pg. 30-31

VANTI, Nadia. Métodos cuantitativos de evaluación de la ciencia: bibliometría, cienciometría e informetría. InvestigaciónBibliotecológica. 2000, 14/29. 9-23.

VESSURI, Hebe (1996) La calidad de la investigación en Venezuela: Elementos para el debate en torno al programa de promoción del investigador. Interciencia. 1996, $21(2)$ 